\title{
失踪的镧系元素
}

廋晓萍 ${ }^{8}$, 朱亚先 ${ }^{*}$

厦门大学化学化工学院, 福建 厦门 361005

摘要: 镧系元素是第 57 号元素锞到 71 号元素镥 15 种元素的统称。它们的化学性质相似, 在生产、生活中起着重 要的作用。本文假想镧系元素失踪后地球发生的一系列变故，借此体现它们在人类生活中的重要性。

关键词: 锞系元素; 发现; 性质; 应用

中图分类号: G64; O6

\section{The Lanthanides Are Missing}

\section{Xiaoping Yu ${ }^{\S}$, Yaxian Zhu *}

College of Chemistry and Chemical Engineering, Xiamen University, Xiamen 361005, Fujian Province, P. R. China.

Abstract: The lanthanides include elements having atomic numbers 57 (lanthanum) through 71 (lutetium). They all behave in a similar way in chemical reactions and play an increasingly important role in production and life. This article imagines a series of changes that have taken place on the earth since the disappearance of lanthanides, which shows their importance in human life.

Key Words: Lanthanide; Discovery; Property; Application

\section{1 锞系元素的失踪}

多多从睡梦中醒来，打开电视，看到新闻在不停地滚动播报：

“今天早上七点, 科学家发现地球上的镧系元素正在消失, 原因不明。镧系元素的失踪将会对 通信、医疗、石油工业等多方面造成严重影响。现已宣布进入紧急戒备状态，请大家不要恐慌……”

这时, 显示屏突然闪烁一下, 新闻主播焦急的声音仍然回响在房间, 电视画面却像是蒙上了一 层纱，什么也看不清。

这是怎么回事？多多突然想起，镧系元素可以突出显示屏的色彩。镧系元素失踪，电视画面自 然就模糊不清了。多多在柜子里找到了说明书, 果然, 上面写着电视的 OLED 显示屏的发光层材料

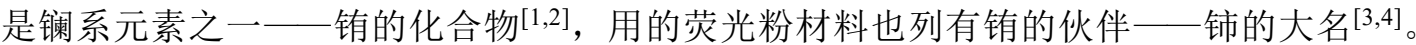

多多打开电脑, 页面却显示网络未连接。这又是怎么回事? 她赶忙在书柜里找到了一本锞系元 素的读物。

原来, 我们的互联网是靠光纤传输信号的, 光纤是一种由玻璃制成的纤维, 重要成分之一就是 氟化锞。多多继续往下读, 发现制造光隔离器需要钝镓石榴石, 光纤放大器需要镨、铒, 光纤传感

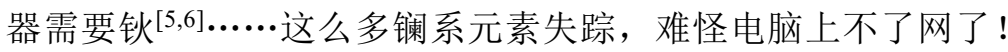

收稿：2020-09-08; 录用: 2020-09-15; 网络发表：2020-09-21

“通讯作者, Email: yaxian@xmu.edu.cn

${ }^{\S} 2019$ 级本科生

基金资助: 国家基础科学人才培养基金(J1310024) 
多多看着空白的网页, 一筹莫展。然而, 更大的乱子还在后头。镧系元素莫名其妙人间蒸发, 给地球这个小行星带来了接二连三的麻烦。

\section{2 无法发动的汽车}

多多今天要去医院看望生病的妈妈, 她刚出小区, 发现街道停着长串的汽车, 后面的司机不停 按着喇叭。

“这是怎么了？”多多好奇地问。

一辆汽车缓缓降下挡风玻璃, 里面的司机探出头来, “小姑娘, 你不知道, 今天早上什么失踪 后, 很多汽车无法启动, 交通全痽疾了! ”

副驾驶位坐着一个与多多年龄相仿的女孩, 她无奈地说道: “爸爸, 是镧系元素失踪了! 很多 汽车电机都是由稀土永磁材料制成, 如钕铁硼永磁体、钐钴永磁体等 ${ }^{[7]}$ 。镧系元素在其中都是不可 或缺的核心。现在电机无法启动, 这些汽车自然也难以行驶了。”

“除此之外, 镧系元素中铈的失踪还带来了另外两件麻烦事儿。铈是汽车尾气催化剂中必不可 少的成分, 它和铂铑这些贵金属一起将一氧化碳、氮氧化合物和未完全燃烧的烃类转化为无毒的二 氧化碳、水和氮气 ${ }^{[8]}$, 可以说是减少大气污染的大功臣。”

“同时, 铈也作为添加剂被大量应用于汽车玻璃中。它可以吸收紫外线和红外线, 不仅起到降 低车内温度的作用, 更保护我们远离了皮肤癌等种种疾病 ${ }^{[9]}$ 。”

“看来, 镧系元素的确很重要啊! ”多多感叹道。

\section{3 乱成一团的医院}

多多到达医院后, 发现放射科前排起了长队, 患者们拿着单子, 唉声叹气。一位患者告诉她, 是放射科的设备出了问题。那么, 这也和镧系元素有关吗?

“没错。”一旁的医生听到他们的对话, 回答道, “钝的失踪让我们苦恼极了。骨折病人拍 X 光片用到的荧光增感剂是添加了三价琙的硫代氧化钝。核磁共振成像中, 我们也会用钝对比剂来加 强扫描 ${ }^{[10]}$ 。现在这些检测都难以进行, 实在是件愁人的事。”

多多继续向前走, 发现泌尿科门口聚集了不少人, 一位医生正通知病人们今天的结石手术取消。

原来, 人们通常靠体外冲击波来治疗结石。但肾结石、输尿管结石和膀胱结石由于位置特殊, 体外冲击波的本领无处施展, 需要钬激光器的帮助 ${ }^{[11]}$ 。钬的失踪导致结石手术不得不暂缓了。

不仅如此, 钬激光器还可以用于切除组织, 给血管止血。它的同伴铒也是个重要角色。铒激光 器能量低于钬, 不能碎石, 却能切除软组织, 特别用于口腔治疗和白内障手术中, 曾给无数白内障 病人带来了光明 ${ }^{[12]}$ 。

多多若有所思, 她穿过人群, 途经肿瘤科, 听见里面的医生正在焦急地讨论, 他们科室看起来 也遇到了不小的麻烦。

“唉, 镧系元素可是放疗的大功臣, 它们辐射轻, 对人体的危害小。镥还被称为癌症克星, 现 在钐-153、铥-170、镥-177 失踪 ${ }^{[13]}$, 放射治疗只能暂缓了, 真是一场灾难......$”$

多多走进妈妈的病房，主治医生这时正巧也在房间，多多向他们讲述了今天的所见所闻。

\section{4 各行各业的麻烦}

听完多多的话, 大家都叹口气。

“哪只是医院呢, ” 护士说, “我们家孩子在野外露营, 不知道为什么, 打火机和火石都点不 着了, 现在正在山上冻得发抖呢。”

“这是因为打火机里的火石是铈铁。” 多多说, “原本齿轮摩擦, 镧系元素燃烧, 迸出火花便 能点燃液态丁烷。如今铈、镧、钕、镨这些元素失踪, 打火机便点不着火了。” 
“核电站也不得不停运了，”妈妈补充道，“我听同事说，是反应堆控制棒的问题。钝的同位 素钝-155 和钝-157 在所有元素同位素中, 热中子吸收截面最大, 因此被应用在钛制轻水堆和快中子 繁殖堆中。除钝外, 钐、镝也是强效的中子吸收器, 用来控制核反应速度 ${ }^{[14]}$, 现在镧系元素失踪, 核电站也只好停运了。”

“我是钢铁行业的, ”隔壁床的病人也加入了谈话, “镧系元素容易和氧、硫、铅等元素化合 生成熔点高的化合物, 这些元素填补在晶粒缺陷中, 生成阻碍晶粒继续生长的膜, 从而使晶粒细化, 显著提高钢的耐磨性、耐腐蚀性和韧性 ${ }^{[13]}$, 是十分重要的工艺。如今镧系元素失踪, 对我们也是不 小的损失。”

“还有储氢材料, ”另一位病人说, “稀土储氢材料咜存密度大于液氢, 体积却只有普通钢瓶 的六分之一, 是一种更为简便易行的理想储氢方式。在大容量电池领域, 应用镧镍合金的镍氢电池 具有很大的发展空间 ${ }^{[13]}$ 。镧系元素对我们产业也是至关重要。”

“远不止这些呢, ”主治医生连叹了好几声, “摄影摄像的镝灯报废了, 单反需要的镧系光学 玻璃使用不了, 石油催化裂化至关重要的镧系催化剂失效 ${ }^{[14]}$......各种各样的麻烦事接踵而至, 各行 各业的人们面临数不尽的困难，谁也不知道该怎么办好。

\section{5 多多与镂的会面}

回到家后, 多多坐在桌前, 一边看书上有关镧系元素的知识, 一边陷入深思。镧系元素加上锌、 钇共 17 位被称为稀土元素, 但其实一点也不 “稀” , 更不是 “土”，只是历史上一个美丽的误会。 它们在地壳中丰度有万分之一，甚至超过了常见金属铜、铅、锌、锡呢 ${ }^{[14]}$ 。

现在，镧系元素为什么失踪了? 人们以后的生活会怎么样呢? 多多想着想着便陷入了梦乡。

梦中她来到一座矿山, 工人们正在热火朝天地采矿。这时, 突然有只金属般冰凉的手从身后拉 住她。

“你是谁？”多多惊讶地睁大双眼，看到一个全身银白的小孩。

“我是镧, 也就是元素周期表的 57 号元素。你可以叫我小镧。”

多多一把抓住她：“到底发生了什么, 你们为什么会失踪呢? 大家都急坏了。”

小镧笑了笑, “别着急, 你先看看这个地方。””

“这是 1787 年瑞典首都附近的伊特比村。别看它只是一个小村子, 我的三个弟弟妹妹一一铒、 琙、镱都是以这个小村庄的名字命名的。”

“这些工人在矿中开采出了一种未知的黑色矿石。1794 年, 芬兰化学家加多林在这类未知矿物 中分离出了一种新的氧化物, 给它起名钎土。后来, 其他化学家又从 “钇土' 中提取了好几种稀土 矿物 ${ }^{[14]}$ 。这是人类发现镧系元素的源头。”

“目前, 世界上发现的稀土矿物有两百多种, 具有工业价值的有五十到六十钟, 其中具有开采 价值的只有十种左右。我再考考你，你知道世界上已探明稀土储量最多的地方在哪吗？”

多多不假思索, “是我们中国内蒙古的白云鄂博！”

小镧笑道, “是的。世界稀土资源分布不均, 中国、美国、俄罗斯、加拿大、澳大利亚等是稀 土储量比较丰富的国家, 中国探明的储量居世界之首。现在中国生产的高纯度稀土已占世界产量的 $80 \%$ 以上, 比较著名的产地有白云鄂博稀土矿、山东微山稀土矿、冕宁稀土矿等。中国是世界上最 大的加工产品出口国[15]。”

见多多点头，小镧又道，“走吧，我带你去见我的弟弟妹妹。”

\section{6 难以区分的家族}

眼前的画面一转, 小锞带多多来到了一个聚会。“那是我的弟弟妹妹们, ”小镧指着和它长相 相似的元素们说, “我们个性十分相近, 里面最像的就数镨和钕了, 历史上, 人们一度把它们当一 
种元素, 直到 1885 年, 奥地利化学家卡尔将两者成功分离, 它们才拥有了自己单独的名字 ${ }^{[14]}$ 。”

“好神奇啊, 就像 15 位多胞胎? ”多多问。

“恩, 这是我们电子排布的特殊性导致的。”小锞笑道, “随着原子序数的增加, 我们依次增 加的电子主要排在了内层的 $4 f$ 能级上。由于位于内层的 $4 f$ 轨道屏蔽能力强, 随着原子序数的增加, 有效核电荷增加较少。通俗说, 虽然我们的父亲——原子核越来越强势, 但是我们的母亲聪明地缓 解了父亲的力量, 价电子受到的 “掌控” 变化不大, 因此我们兄弟姐妹的长相、性格比较相近。但 是, 毕竟不是同父同母的多胞胎, 仔细看还是有不少差异哦! 你看, 我们个头的总趋势是减小的。”

“我知道！”多多抢着说: “这就是著名的镧系收缩 ${ }^{[16]}$ 。”

“没错。我和老十五镥, 个头就差 $14 \mathrm{pm}$, 性格也不太一样。你再看销和镱, 它们的个头比相邻 兄弟大得多, 这是因为它们的 $4 f$ 轨道电子是半充满或全充满的, 这种结构能更好地屏蔽来自父亲的 掌控, 身体就更舒展了呢。”

“镧系收缩还对我们后面的元素有影响, 如钼和铇、铌和钽、锆和铪的个头差不多, 是三对形 影不离的好朋友。此外, 镧系收缩也使钇的离子半径和我们家族的铒、钬相近, 这也是钇爱同我们 一起出现的原因 ${ }^{[16] 。 ” ~}$

多多说: “我明白了。你们这么相似, 又喜欢居住在一起, 难怪人们很难将你们分离。”

“虽然难, 但是人们还是找到了很多办法, 比如氧化还原法、溶剂萃取法、离子交换法等, 这 些分离手段使我们的应用大大拓展了[16]。”小镧道。

这时, 突然有元素兴奋地大喊起来。

小针朝那边望了一眼, “氧来参加舞会了, 我可以给你露一手! ”

\section{7 镧系元素的性质}

在化学国度中, 氧可谓人缘最好的元素, 除了向来高冷的稀有气体, 几乎每个元素都同氧交好。 随着欢快的乐曲声响起, 小镧和氧走到舞台中央, 轻盈地旋转起来, 随着舞步加快, 四周浌开火花, 美丽极了。

多多不由坚起大拇指: “跳得真好!”

小镧腼腆地笑了笑, “谢谢! 不只是我, 我们家族的铈、钕都会这样的舞蹈。人类正是利用这 一点, 制成了火石等物品。除氧之外, 我们也能和氮与氯的单质发生类似的反应呢。”

“你们待在周期表的下面, 离大家那么远, 是不是有点孤独啊? ”多多好奇地问。

“不会, ”小镧摇头说, “我们的朋友很多, 虽然住得远, 但是大家经常走动。我们十分好客, 化学活性与第二主族的碱土金属相近, 喜欢把电子送给别人或与他人分享。除了与非金属反应, 我 们还可以和绝大部分金属形成金属间化合物, 而且这些金属间化合物大多都具有特殊的性能。比如 我们与铁族形成的化合物具有永磁性能, 与镍形成的化合物具有强烈的吸氢性能, 与镁等有色金属 形成的化合物具有良好的力学性能 ${ }^{[14]}$ 。这样看来, 我们在周期表中算比较受欢迎的家族呢。”

多多点点头。

“来, 一起喝下午茶吧。”小镧提议道。

\section{8 神奇的色彩与磁性}

镧系元素们面前摆的下午茶有趣极了, 从 $\mathrm{La}^{3+}-\mathrm{Lu}^{3+}$ 摆成了 $\mathrm{U}$ 型, 钝离子在 $\mathrm{U}$ 型底部坐镇, 其 他离子分列两旁, $U$ 型左侧的颜色依次为无色、绿色、红色、紫色、浅黄色、浅紫色, U 型右侧的 颜色依次为浅紫色、浅黄色、褐色、红色、绿色, 最后到无色 ${ }^{[14]}$, 不仅色彩斑斓且十分对称, 看起 来交相呼应，仿佛一圈彩虹。

铒向多多介绍, “这些下午茶都是我们的离子颜色, $4 f$ 轨道电子喜爱串门, 容易发生 $f-f$ 跃迁, 电子在跃迁时吸收了部分可见光的能量, 我们的离子就呈现出了相应互补光的色彩。” 
小镧补充道: “随着原子序数增加, 电子有规律地填充, 因此, 我们的颜色变化相当有规律。”

多多仔细地看了一圈, “瞧, 个别元素多吃多占, 有两杯茶哦。”

铒笑道, “呵呵, 这是个人能力所致。我们属于 IIIB 族, 大多数兄弟姐妹习惯失去外层的三个 价电子, 成为三价离子, 所以只有一杯茶。但是, 由于电子层的构型缘故, 有的元素可以呈现两种 价态。拿铈说吧, 它面前无色的是三价离子, 橙黄色的是四价离子。铕呢, 除了浅紫色的三价离子, 还有草黄色的二价离子[14]。”

多多不好意思地笑了。铒继续说: “由于我们的电子能级极为丰富, 因此具有复杂的光、电、 磁等性能。人们也将我们用于制造彩色玻璃: 加入钕可使玻璃成酒红色, 加入镨可使玻璃变成绿色; 我嘛, 可使玻璃变成粉红色……这些彩色玻璃色泽变幻莫测, 美丽非凡 ${ }^{[13]}$ 。”

多多惊叹一声。

这时, 音乐响了起来, 元素们开始翩翩起舞。钕拉着舞伴, 旋转起来, 最后越旋越高, 飞到了 空中…...

“奇怪！竟然没有掉下来？”多多惊讶地问。

镨笑着说: “这是我们的磁性导致的, 磁性可以说是我们家族最重要的特征之一, 钕是我们之 中最杰出的一个, 它制成的钕磁铁一克能够吸引起两公斤的铁呢！人们将我们制成的稀土永磁材料 称为永磁之王。我们的纳米磁性材料用于磁存储器、磁流体、巨磁阻, 可以使性能提高、器件小型 化。此外, 我们的磁性材料也被应用在微波通讯、仪器仪表、医疗交通等多个领域, 在高新技术材 料方面的应用尤其引人注目 ${ }^{[14]}$ 。”

镨拿出一个小球, 给多多示范。它将小球放在手心, 再一翻, 小球没有掉落, 仍然牢牢贴着。 多多好奇地凑上前, 碰了碰悬空的小球, 谁知球一下子掉落在地上。

“对不起, ”多多讪讪道, “我太不小心了。”

“不是你的错。” 镨说, “是我的居里点太低了。我的磁铁在 30 摄氏度就会失去磁性, 远低于 你身体的温度, 所以你一碰小球就掉了。”

\section{9 尾声}

快乐的时光总是过得很快, 分手的时候到了。临别前, 多多又提及了心中的疑惑： “我已经明 白了镧系元素对人类的意义。你们就好比维生素, 虽然量少, 但作用巨大。人们已经离不开你们了! 但是你们为什么会失踪呢？你们还会重新回来吗？”

小镧温和地看着她。

“诚然, 你们需要我们。可是需要并不代表一味索取。近些年人类大肆开采稀土矿, 给自然带 来了许多伤害和环境污染。再者, 我们在地壳中的含量虽然不少, 能开采的资源却不多, 而资源的 浪费又非常惊人……”

多多怔怔地看着它，小镧继续说:

“倘若继续下去, 也许你今天看到的灾难有一天会成为现实。万物有灵皆需爱, 天地无情不可 知。常将有日思无日, 莫待无时思有时。”

多多看着小镧, 若有所思。

“多多, 醒醒! ”

多多睁开眼睛, 发现自己正躺在床上, 妈妈没有住院, 镧系元素也没有失踪, 一切都井然有序 地进行着。

原来一切只是一场梦啊!

或许一切也不只是一场梦。 
[1] 刘嵩. 稀土销配合物 OLEDs 特性的研究[D]. 天津: 天津理工大学, 2006.

[2] 辛琦. 基于稀土铺配合物的有机电致发光器件性能研究[D]. 吉林: 中国科学院长春光学精密机械与物理研究所, 2007.

[3] 刘跃. 稀土信息, 2009, No. 6, 30 .

[4] 赵文卿, 关荣锋, 王杏. 无机盐工业, 2008, No. 10, 15 .

[5] 李蓥德. 稀土信息, 1997, No. 1, 11 .

[6] 何钰泉, 关铁樑. 科学通报, 1984, No. 7, 18.

[7] 叶萧然. 铸造技术, 2015, No. 1, 32.

[8] 郭䎣. 中国稀土学报, 2007, No. 1, 4.

[9] 张晓伟, 李梅, 柳召刚, 胡艳宏, 吴锦绣. 硅酸盐通报, 2009, No. 6, 108.

[10] 苏铭. 稀土元素. 北京: 清华大学出版社, 2000.

[11] 何家杨. 尿石防治. 上海: 复旦大学出版社, 1998.

[12] 谢品华, 刘文清. 光电子技术与信息, 1997, No. 4, 5 .

[13] 本刊讯. 首都食品与医药, 2018, No. 9, 5 .

[14] 叶信宇. 稀土元素化学. 北京: 治金工业出版社, 2019 .

[15] 窦光宇. 百科知识, 2009, No. 7, 29.

[16] 刘欣锦, 朱亚先, 高飞. 无机元素化学. 北京: 科学出版社, 2008. 